\title{
The Magnetic Nanoparticle Movement in Magnetic Fluid Characterized by the Laser Dynamic Speckle Interferometry
}

\author{
Xijun Wang \\ Changchun Institute of Optics, Fine Mechanics and Physics, State Key Laboratory of Applied Optics, Chinese Academy of Sciences, \\ Changchun 130033, China
}

Correspondence should be addressed to Xijun Wang; xjwang@ciomp.ac.cn

Received 16 July 2013; Revised 25 December 2013; Accepted 9 January 2014; Published 24 February 2014

Academic Editor: Rakesh Joshi

Copyright (C) 2014 Xijun Wang. This is an open access article distributed under the Creative Commons Attribution License, which permits unrestricted use, distribution, and reproduction in any medium, provided the original work is properly cited.

A dual scanning laser speckle interferometry experiment was designed to observe the dynamic behavior of the magnetic fluid actuated by a magnetic field. In order to improve the spatial resolution of the dynamic speckle measurement, the phase delay scanning was used to compensate the additional phase variation which was caused by the transverse scanning. The correlation coefficients corresponding to the temporal dynamic speckle patterns within the same time interval scattering from the nanoparticles were calculated in the experiment on nanoscale magnetic clusters. In the experiment, the speckle of the magnetic nanoparticle fluid movement has been recorded by the lens unmounted CCD within the interferometry strips, although the speckle led to the distinguished annihilation of the light coherence. The results have showed that the nanoparticle fluid dynamic properties appeared synergistically in the fringe speckles. The analyses of the nanoparticle's relative speed and the speckle pattern moving amount in the fringes have proved the nanoparticle's movement in a laminar flow in the experiment.

\section{Introduction}

A magnetic fluid is a colloidal suspension of the paramagnetic nanoparticles in a liquid carrier which can be easily actuated by applying a magnetic field [1]. Therefore the application areas have been widened into the nanotechnologies such as nanogenerators, nanopumps, and nanomotors, and many other nanodevices with a variety of magnetic fluids have been coming of age [2-4].

The dynamic property of magnetic fluids in combination with high absorption coefficient to visible light has been found useful in optical device applications such as information displays and light modulators [5-8].

Because the performance of magnetic-fluid-based optical devices will depend on the movement of magnetic fluid responding to the applied magnetic field, it is significantly crucial to characterize the behavior of the magnetic particles in a magnetic field. However, the dynamics of magnetic particles in magnetic fluid, unlike the nanoparticles fixed in a solid bulk material, is difficult to characterize by adopting the conventional methods such as AFM and SEM. Instead, it is possible to use noncontact and nondestructive method based on the laser speckle technique [9-12].

Although the light scattering method has discovered applications in the characterizations of the dynamic laser speckle from a magnet fluibuiltd $[13,14]$, the contrast of the speckle or the scattering intensity may open only a general observation to the bulk magnet solution. The magnetic nanoparticles were also covered in the fluid that could not be observed. The average signal of the PMT (photomultiplier tube) or photodiode is too deficient to meet the requirement for the nanoparticle movement in the solution when the exerted magnetic field drives the magnetic particles rotating, aligning, or piling up. So the speckle images captured by CCD image sensor will record an array of optoelectronic data of the speckle in each equal interval.

How to coordinate the speckle movement from a variation of the surface profile resulted in the solution of the moving magnetic particles which could be driven by an electromagnetic tesla coil array. The optical interferometry that the crucial component works in an AFM is the first choice to build up the coordinates in the speckle images. 


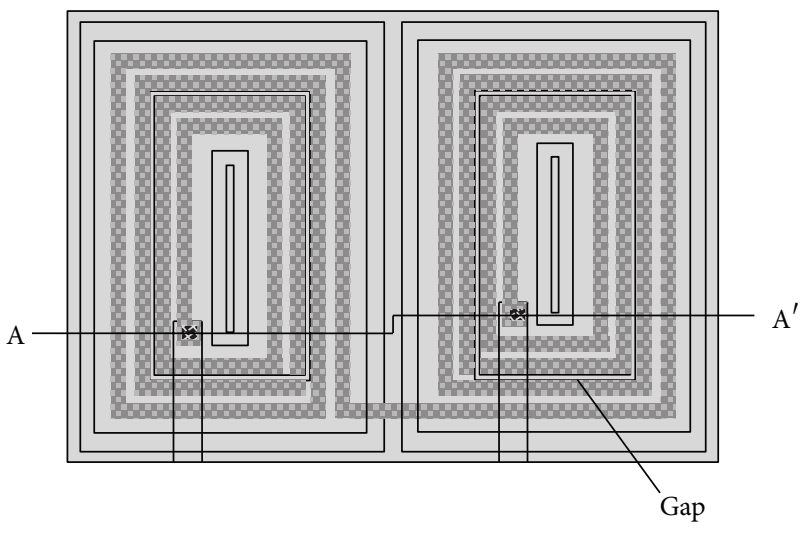

(a)
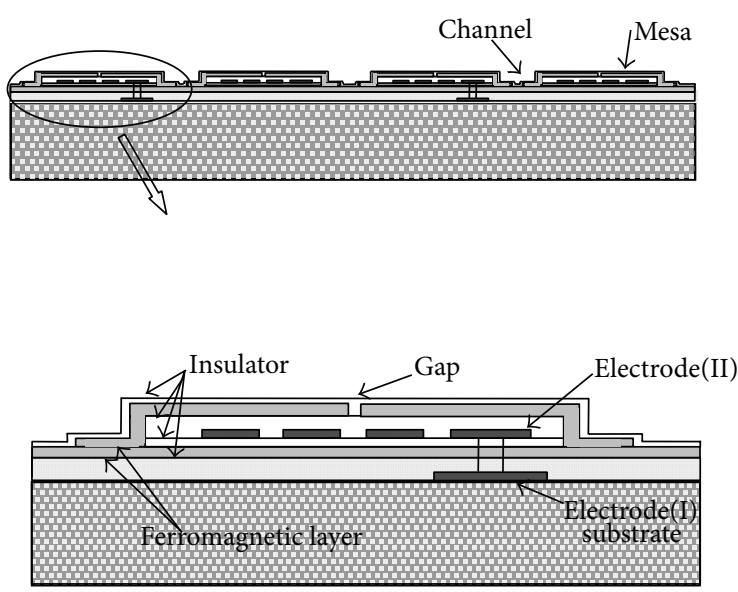

(b)

FIgURE 1: (a) Top view of the device with the spiral electrode coils and the rectangular magnetic gaps. (b) Cross-sectional view of the device with a magnified view to the vertical layer structure.

In order to make stationary interferometry strips, the two questions should be answered that the higher-order interferometer speckle decreases the coherence of the scattering light so significantly that the strips could not be found; while the transverse scanning find a uniform view field, the nonlinear additional phase should be compensated by the phase delay to make the strip "still."

While a dual scanning laser speckle interferometry to observe the dynamic behavior of the magnetic fluid actuated by a magnetic field was built up, the experimental results were discussed to characterize the movement of the granules in the magnetic fluid.

\section{Experimental Setup}

2.1. Magnetic Fluid and the Device. In the experiment, magnetite $\mathrm{Fe}_{3} \mathrm{O}_{4}$ nanoparticles were from the $\mathrm{F}^{2+}$ and $\mathrm{F}^{3+}$ precipitation with the $\mathrm{F}^{2+} / \mathrm{F}^{3+}$ molar rate $1: 1.8$, acting temperature $80^{\circ} \mathrm{C}$ [15], and keeping $\mathrm{pH} 9 \sim 10$ by gradually dropping the concentrated ammonia into the agitation solution. The magnetite $\mathrm{Fe}_{3} \mathrm{O}_{4}$ particles were separated by a magnet directly. The average particle size was around $40 \mathrm{~nm}$ by AFM and the saturation magnetization was around $70 \mathrm{emu} / \mathrm{g}$. The magnetite particles were coated with sodium oleate keeping $\mathrm{pH} 5 \sim 6$ and dispersed in n-nonane $\left(\mathrm{C}_{9} \mathrm{H}_{20}\right)$, which was one clear ingredient of commercial kerosene.

The operation of a magnetic fluid display device relied on the modulation of the thickness of a magnetic fluid layer which was placed on the substrate, and the characterization was focused on a magnetic fluid actuator to form a pixel of a display device. The actuator consisted of two units each with an area of $1.5 \times 0.5 \mathrm{~mm}^{2}$ formed by a thin layer of ferromagnetic thin film enclosing a spiral conductor electrode which could be manipulated easily by controlling the current going through the spiral coils in Figure 1(a) [8]. The channel stored the magnet fluid and formed the effective path to the mesa. The length of the channel was $1.5 \mathrm{~mm}$, and its width was the same as the gap in the mesa, so the width was 15 micron in the design.

Most of the magnetic fluid would initially stay in the channel around the mesa due to the capillarity when no electric field was applied, and only a minute thin solution layer of the liquid would reside on top of the mesa area in Figure 1(b).

The thickness of the magnetic fluid was modulated by the step magnetic field which was generated by the electric current pulse through the circuit underneath the actuator mesa. The magnetic force exerted by the electric field drove the ferrofluids into the region of strong magnetic intensity and made the display pixel darker. When the current was off, on the other hand, the surface tension of the fluid restored the force of the liquid back to the original state and the pixel became brighter, in which the light and dark states of the pixel could be thereby produced correspondingly.

2.2. Experimental Design. The scanning interferometer used a He-Ne laser with the beam collimating, expanding, splitting, and combining to generate the reference and object light to create interferometer patterns practicably. Two nearly parallel light beams, one as the object beam was incident to the magnetic unit containing magnetic fluid and scattered by the magnetic particles to form a speckle field and the other as the reference beam was incident to the speckle field, which generated interferometer fringes in the speckle field.

The transverse scanning device that was a spinning optical parallel plate keeping the incident angle invariable aligned the object light to the pixel mesa by transverse scanning precisely with an additional phase. The phase delay was used to compensate the variation of the additional phase caused by the view field requirement of the object light. The experimental setup was shown in Figure 2.

Adjusting the angle of the two light beams could change the interferometer fringe spacing and the fringe contrast in the laser speckle field. Assuming that the object light and 


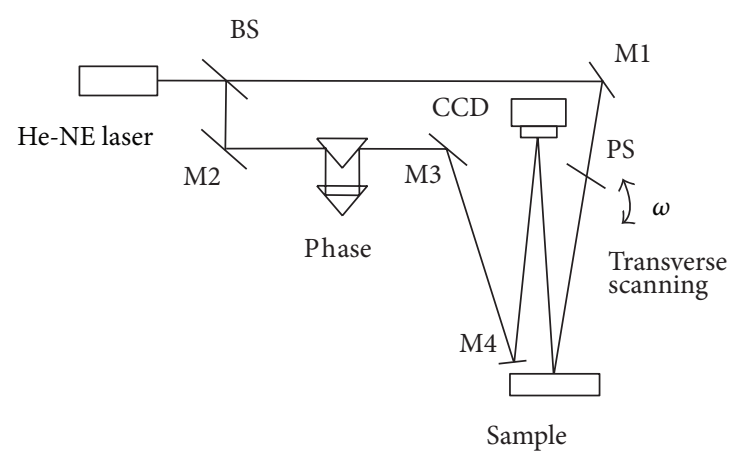

FIGURE 2: Schematic of a dual-scan laser speckle interferometer experiment.

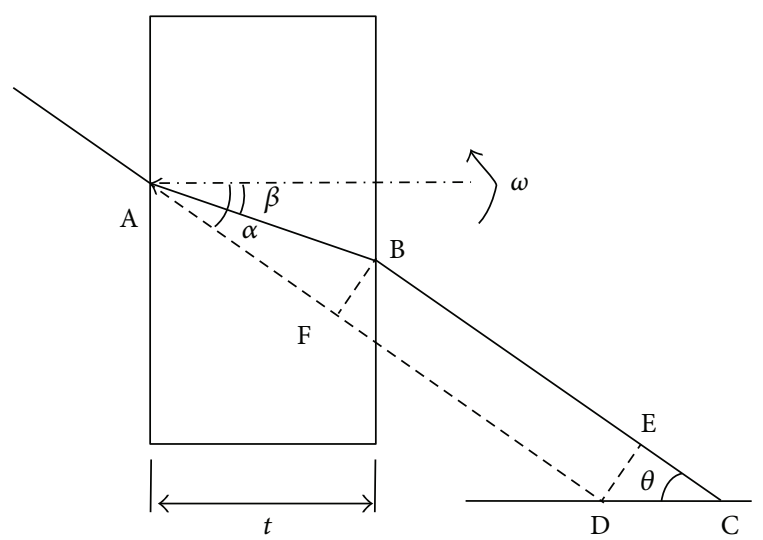

FIGURE 3: Transverse scanning plate to subjoin phase delay.

reference light were in the same incident angle $i$ and symmetrically incident to CCD, the spacing of the interferometer fringe was given by

$$
\Delta x=\frac{\lambda}{2 \sin i} .
$$

2.3. Phase Compensation. An additional phase shift was into the object light path while using a transverse scanning plate as shown in Figure 3, where $\alpha$ was the incidence angle of the plate and $\beta$ was the refraction angle in Snell's law: $n \cdot \sin \beta=$ $\sin \alpha$, where $n$ was the refractive index of the plate and the air refractive index was 1.0.

The optical path length $L$ from the incidence point A that was the spinning center on the transverse scanning plate to the point $C$ on the sample surface was given by

$$
\begin{gathered}
L=n \mathrm{AB}+\mathrm{BC}=n \mathrm{AB}+(\mathrm{AD}-\mathrm{AF}+\mathrm{CE}), \\
\mathrm{AB}=\frac{t}{\cos \beta}, \\
\mathrm{AF}=\mathrm{AB} \cdot \cos (\alpha-\beta), \\
\mathrm{CE}=\mathrm{AB} \cdot \sin (\alpha-\beta) \cot \theta,
\end{gathered}
$$

where $\theta$ was the incident light angle of the ferrofluids sample surface. Thus, the optical path length could be written as

$$
L=\mathrm{AD}+\{n-\cos (\alpha-\beta)+\sin (\alpha-\beta) \cot \theta\} \cdot \frac{t}{\cos \beta},
$$

where $\mathrm{AD}$ and $\theta$ were invariants in the experimental setup. The derivative of $L$ versus $\alpha$ which gave the changing rate of the optical path length varied with the spinning angle of the transverse scanning plate. Consider

$$
\begin{aligned}
\frac{d L}{d \alpha}= & \frac{t \sin \beta \cos \alpha}{n \cos ^{3} \beta}[n-\cos (\alpha-\beta)+\sin (\alpha-\beta) \cot \theta] \\
& +\frac{t(n \cos \beta-\cos \alpha)}{n \cos ^{2} \beta}[\sin (\alpha-\beta)+\cos (\alpha-\beta) \cot \theta] .
\end{aligned}
$$

The rotation of the transverse scanning plate was driven by a stepper motor with a miniature step angle $0.009^{\circ}$. OPD (optical path difference) from transverse scanning could be obtained by formulas (3) and (4). The OPD curve was showed in Figures 4(a) and 4(b), while the thickness of the parallel plate is $0.5 \mathrm{~mm}$.

The transverse scanning could be controlled in half wavelength in the setup and Figure 4(a) showed that the tendency of the OPD varied with the spinning angle depending on formula (4) which is symmetrical with the spinning angles and nonlinear in the angle range. The ferrofluids incident angle $\theta$ became smaller and the OPD varied faster. So the incident angle was around $2^{\circ}$ in the experiment.

Nearby the spinning angle zero location, the curve became linear. But some light might have reflected back the laser source and made some noise in the zero angle, so in the experiment, the transverse scanning would deviate a little more to get the higher resolution in tens nanometers.

The optical path of the reference light was adjusted using a $180^{\circ}$ prism phase compensation device driven by another stepper motor in a straight line reciprocating motion. The OPD created by the phase delay was twice the movement distance.

For a given incidence angle $\alpha$ of the transverse scanning plate, a certain amount of phase compensation was to be determined so as to determine the rotation angle of the phase delay step motor and also to synchronize the phase delay with the transverse scanning by creating the driving parameters for both of the step motors controlled by microcomputer units.

\section{Experimental Results}

$\mathrm{He}-\mathrm{Ne}$ laser with an output power of $3 \mathrm{~mW}$ at $632.8 \mathrm{~nm}$ was used as the light source, and a magnetic fluid film with a thickness of about $20 \mu \mathrm{m}$ was used as the device for test. The magnetic fluid display cell was driven by step rectangular pulses with a duty ratio of $1: 2$ and a pulse width of $100 \mathrm{~ms}$. The response time of the device was measured to be around $400 \mathrm{~ms}$ with a driving current of $70 \mathrm{~mA}$. The device was placed horizontally in the optical path.

To get an unlimited localization of the speckle image, the unmounted lens CCD and the free space propagation model 


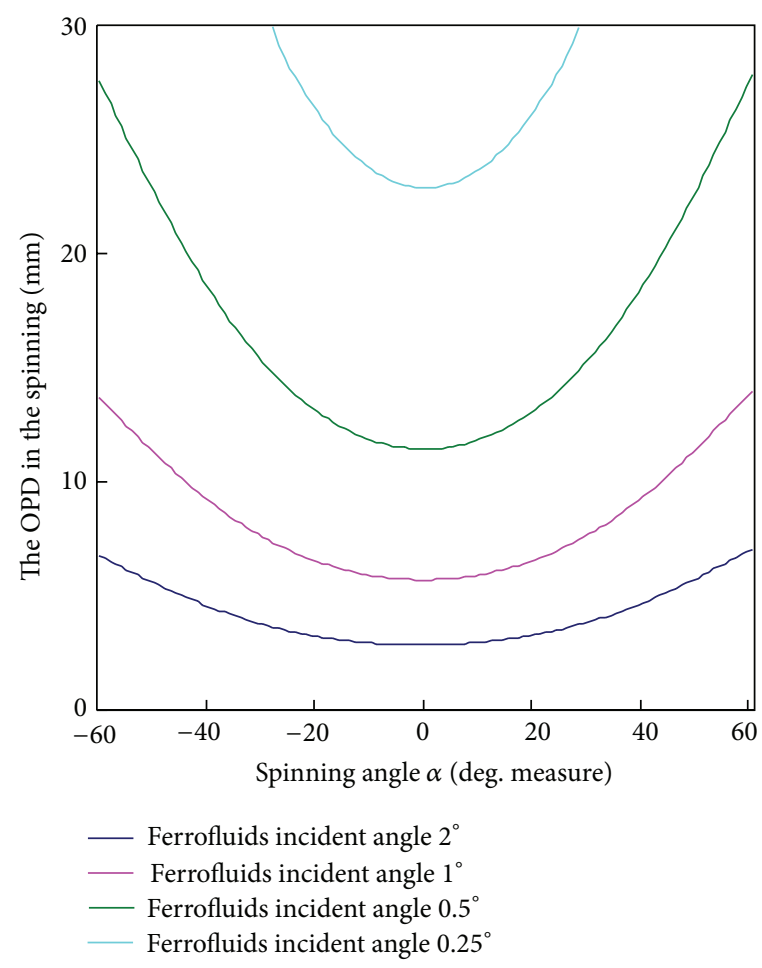

(a)

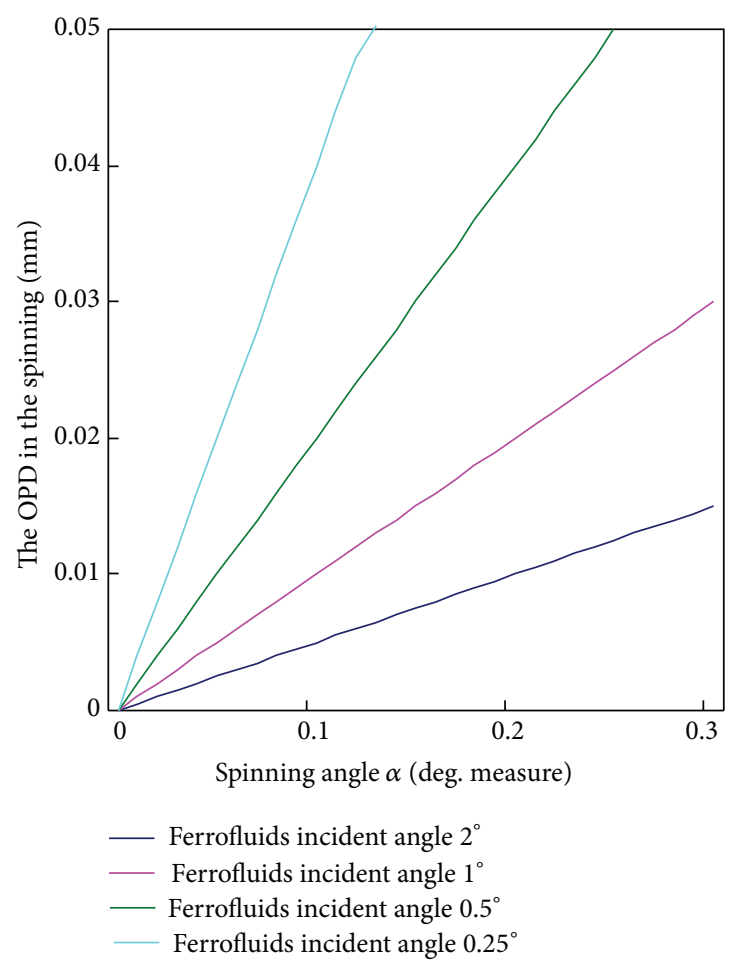

(b)

FIGURE 4: The OPD induced from the transverse scanning in all angle range; (b) was the OPD linear in small angle.

were adopted. The CCD was placed at $30 \mathrm{~cm}$ vertically above the sample surface.

The energy ratio between the object and the reference light was adjusted to balance each other using polarizers in order to produce visible interferometer fringes.

The speckle patterns were recorded continuously by the CCD camera with a frame rate of $25 \mathrm{fps}$ and an image size of $640 \times 480$ pixels, and the CCD operation was synchronized with the electric current pulse driving the magnetic fluid. While the incident angle in which the light beams shined upon the CCD was one degree, the fringe spacing was estimated to be $19 \mu \mathrm{m}$ by formula (1). The incident angle increased twice; on the contrary, the fringe space decreased twice nearly.

Because the speckle was the highest order coherent of the laser beam, the interferometry fringe would not be easy to capture even if the laser had an excellent coherence. Making the two beams equal was the first step to utilize the polarization method.

After the speckle had appeared in the object, a beam shined on the magnet fluid device and it was necessary to find a zero order fringe by the detailed adjusting phase delay with miniature step driving. In spite of the probably appearing fringes, interferometer strip is difficult to observe in naked sight because of the lesser strip contrast within background noises.

Partly, the unstable fringes were captured with the CCD, until the transverse scanning both stabilized with the magnet particle movement and synchronized with the phase delay.
A set of laser speckle interferometer fringes in the same intervals recorded by the CCD was showed from Figures 4(a) to $4(\mathrm{~b})$.

The thin and crowed strips with an arc were the interferometry fringes reflected by the surfaces of the multiple lenses and became main noises.

The intensity relation correlation coefficient in neighbor named "neighbor coefficient" that means the coefficient calculation as the pairwise correlation. From Figures 5(a) to 5(f), the neighbor coefficient was, respectively, 0.5197, 0.4329, 0.5340, 0.8125, and 0.2175, in Figures 5(a) 5(f) sequence.

The coefficient showed that Figure 5(b) compared with Figure 5(a) began to change as the coefficient decreased; Figure 5(c) compared with Figure 5(b) changed intensively as the coefficient became less; Figure 5(d) compared with Figure 5(c) began to change slowly as the coefficient recovered; Figure 5(e) compared with Figure 5(d) tended to stop the movement as the coefficient became more; Figure 5(f) compared with Figure 5(e) stopped the movement as the coefficient jumped when the driving current of the coil was off.

To study the speckle profile movement, the centerlines and laser speckle interferometer bright fringes were extracted simply by the wavelet denoising, the contrast enhancement, and the binarization algorithm in a MATLAB software. The calculation from Figure 5 is converted to Figure 6.

With the center line of the interferometer, bright stripswere as a calibration, and the lower left corner of the center line from article 1 interferometer fringe calibration to 1 , to 


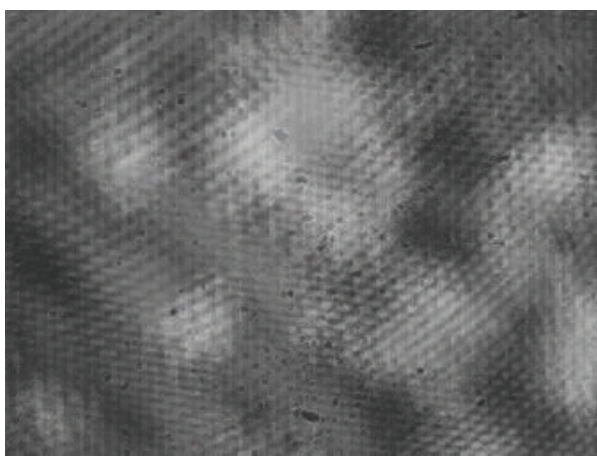

(a)

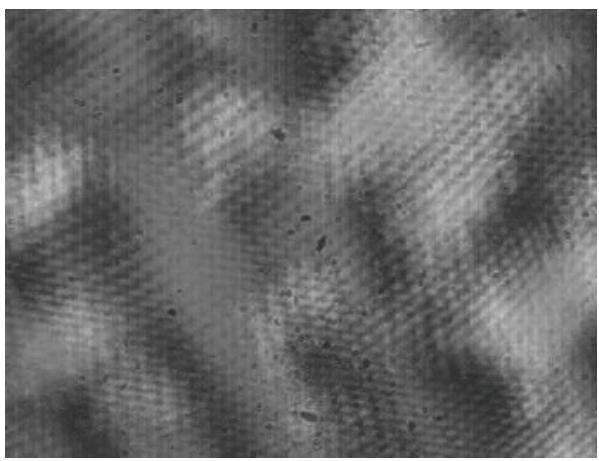

(c)

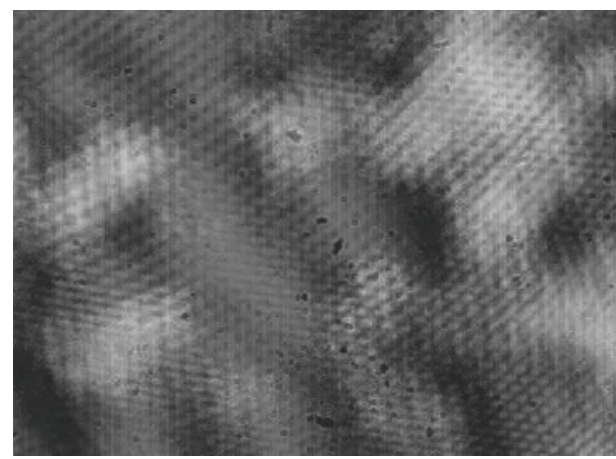

(e)

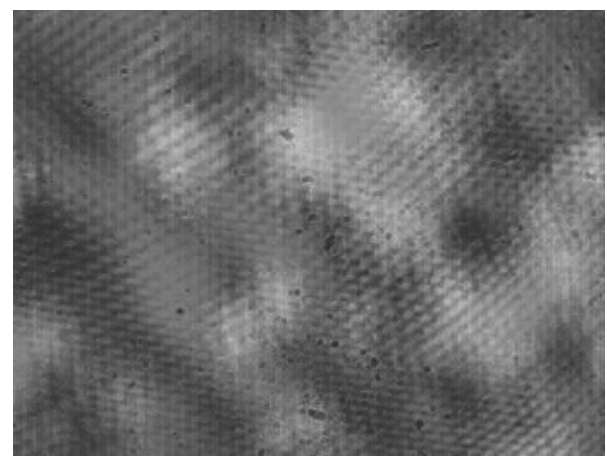

(b)

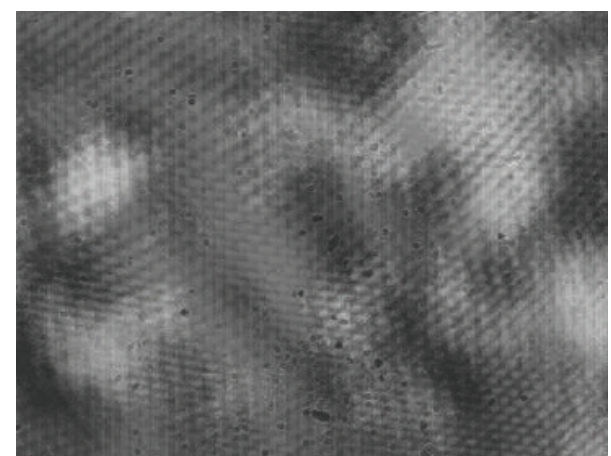

(d)

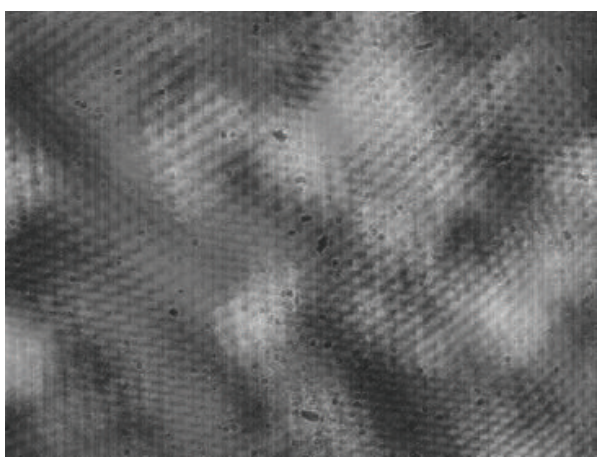

(f)

FIgURE 5: The sequent patterns of the dual-scan laser speckle interferometry.

the upper right corner to the center line of the interferometer fringes in turn calibration for $2,3, \ldots$.

A speckle pattern was identified and circled as a reference for tracking as shown in Figures 6(a) 6(f), where the number next to the circle represents the relative position of the speckle with respect to an interferometer fringe. The black mark in the middle of the reference speckle was the centroid of the speckle. The origin of the graph corresponds to Figure 6(a). The marked number 3.9 in Figure 6(a) was shown as the speckle and strip tracking coordinates.

To calculate the speckle movement from Figures 6(a) to 6(b), the speckle movement was 0.7 strip, and the others were $1.1,1.0,-0.6$, and -1.6 strips. The speckle moved forward or backward in the interferometry strips because the nanoparticles were driven by the coil current in, on, or off state.
The speckle pattern moving amount in the fringes showed that the nanoparticles moved quicker in the piling up than in the quitting in the same time intervals. Therefore, the electromagnetic force was less than the gravity and capillarity to keep laminar flow of the nanoparticle fluid but not the turbulence flow of the solution.

Every strip reflected light expressed twice OPD of one wavelength $632.8 \mathrm{~nm}$, so one strip is related to $632.8 / 2=$ $316.4 \mathrm{~nm}$ variation of the magnetic nanoparticles in the interval 1/25 second. Furthermore, the nanoparticle piling rate is around 7.6 micron per second. The mesa area was $1.5 \times 0.5 \mathrm{~mm}^{2}$ and the effective "channel" area was $1.5 \times$ $0.015 \mathrm{~mm}^{2}$, so the relative speed between nanoparticles in the channel was $0.3 \mathrm{~mm} / \mathrm{s}$. In other words, one step pulse made the nanoparticle move $\sim 0.3 / 25 \mathrm{~mm}$ (12 micron), in which the distance was far less than the path length of the solution. 


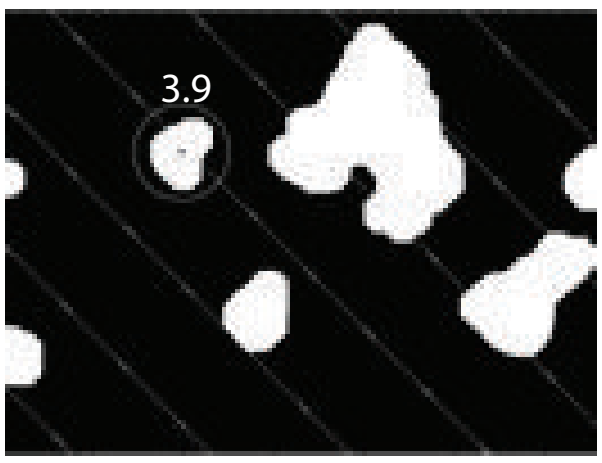

(a)

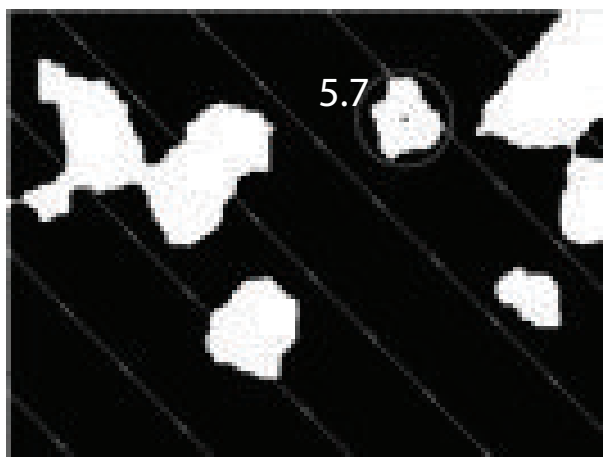

(c)

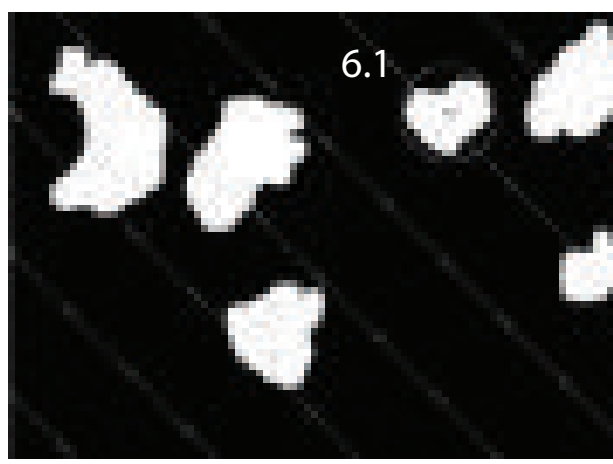

(e)

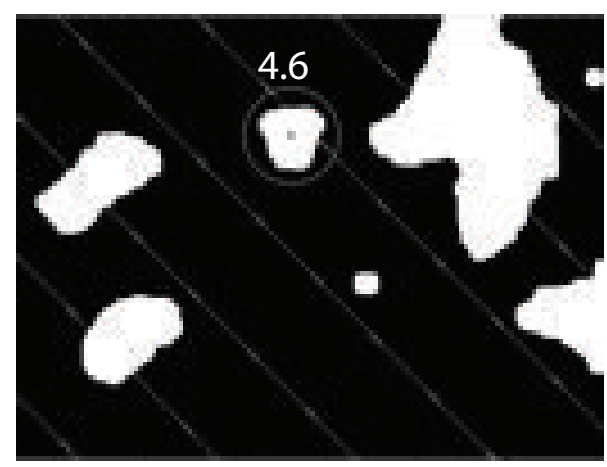

(b)

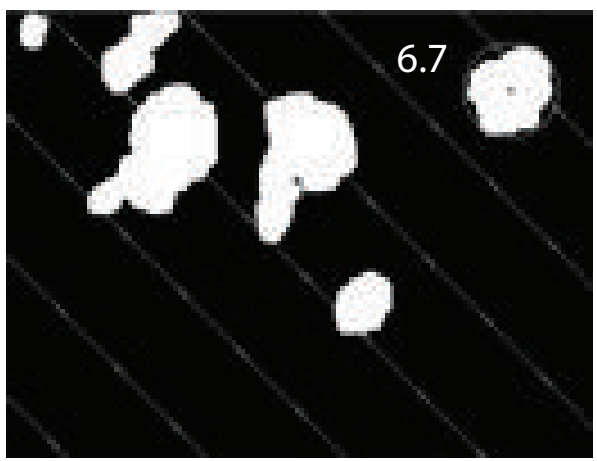

(d)

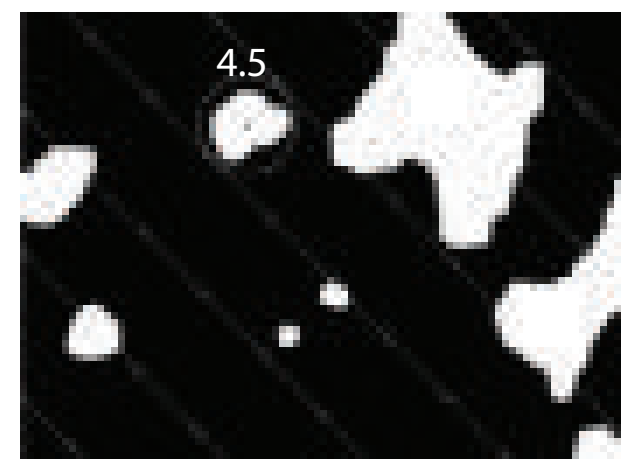

(f)

FIGURE 6: Image binarization and tracking of the speckle pattern movement.

Furthermore, the nanoparticle's relative speed variation also proved that the nanoparticle's movement in the experiment was a laminar flow.

\section{Conclusions}

Although the laser speckle was a higher order coherent phenomenon and the speckle led to the distinguished annihilation of the light coherence, the speckle of the magnetic nanoparticle fluid movement would be recorded by CCD within the interferometry strips. The development of the magnetic nanoparticle fluid characterization focused on a dynamic interaction of the magnet nanoparticles.

It was obvious that the scanning interferometry speckle could capture the nanoparticle and nanostructure's movements, which was different from the light scatting studies which focused on measuring the scattering intensity with a statistical averaged calculation in contrast with the patterns, or with a phase microscope method in a static micron dimension of the speckle patterns $[13,14]$.

The experiment showed that the nanoparticles fluid dynamic properties could synergistically appear in the fringe speckles. The analysis of the nanoparticle relative speed and the speckle pattern moving amount in the fringes proved the nanoparticles' movement in a laminar flow in the experiment.

\section{Conflict of Interests}

The author declares that there is no conflict of interests regarding the publication of this paper. 


\section{Acknowledgments}

The author is fully grateful for the support of the study by the National Science Fund of China (NSFC) (no. 60748026) and Korea Science and Engineering Foundation (KOSEF) (Grant no. F01-2005-000-10169-0).

\section{References}

[1] B. M. Berkovsky, V. F. Medvedev, and M. S. Krakov, Magnetic Fluids Engineering and Applications, Oxford University Press, Oxford, UK, 1993.

[2] M. Zahn, "Magnetic fluid and nanoparticle applications to nanotechnology," Journal of Nanoparticle Research, vol. 3, no. 1, pp. 73-78, 2001.

[3] K. Nakatsuka, "Trends of magnetic fluid applications in Japan," Journal of Magnetism and Magnetic Materials, vol. 122, no. 1-3, pp. 387-394, 1993.

[4] K. Raj, B. Moskowitz, and R. Casciari, "Advances in ferrofluid technology," Journal of Magnetism and Magnetic Materials, vol. 149, no. 1-2, pp. 174-180, 1995.

[5] J.-W. Seo and S. J. Park, "An experimental study of light modulator using magnetic fluid for display applications," Journal of Magnetism and Magnetic Materials, vol. 192, no. 3, pp. 499-504, 1999.

[6] J.-W. Seo, S.-M. Jeon, S. J. Park, and H.-S. Lee, "An experimental and numerical investigation of flat panel display cell using magnetic fluid," Journal of Magnetism and Magnetic Materials, vol. 252, pp. 353-355, 2002.

[7] J.-W. Seo, H. Kim, and S. Sung, "Design and fabrication of a magnetic microfluidic light modulator using magnetic fluid," Journal of Magnetism and Magnetic Materials, vol. 272-276, pp. E1787-E1789, 2004.

[8] J.-W. Seo and X.-J. Wang, "Magnetic-fluid microelectrornechanical light modulator," Optics and Precision Engineering, vol. 13 , no. 5 , pp. 542-547, 2005.

[9] C. A. Thompson, K. J. Webb, and A. M. Weiner, "Diffusive media characterization with laser speckle," Applied Optics, vol. 36, no. 16, pp. 3726-3734, 1997.

[10] R.-S. Lu, G.-Y. Tian, D. Gledhill, and S. Ward, "Grinding surface roughness measurement based on the co-occurrence matrix of speckle pattern texture," Applied Optics, vol. 45, no. 35, pp. 88398847, 2006.

[11] W.-W. Feng, M.-J. Liu, X.-Q. Wang, F.-R. Shi, J. Zhang, and R.$\mathrm{X}$. Jiang, "Feature extraction and recognition of laser speckle for special material surface," Infrared and Laser Engineering, vol. 36, no. 2, pp. 186-188, 2007 (Chinese).

[12] X.-F. Li, J. Xu, J.-J. Luo, L. Cao, and S. Zhang, "Noise analyzing and denoising of intensity image for laser active imaging system," Infrared and Laser Engineering, vol. 40, no. 2, pp. 332337, 2011 (Chinese).

[13] J. Philip and J. M. Laskar, "Optical properties and applications of ferrofluids-a review," Journal of Nanofluids, vol. 1, no. 1, pp. 3-20, 2012.

[14] S. Brojabasi and J. Philip, "Magnetic field dependant backscattering of light in water based ferrofluid containing polymer covered $\mathrm{Fe}_{3} \mathrm{O}_{4}$ nanoparticles," Journal of Applied Physics, vol. 113, Article ID 054902, 2013.

[15] S. Wu, A.-Z. Sun, F.-Q. Zhai et al., " $\mathrm{Fe}_{3} \mathrm{O}_{4}$ magnetic nanoparticles synthesis from tailings by ultrasonic chemical coprecipitation," Materials Letters, vol. 65, no. 12, pp. 1882-1884, 2011. 

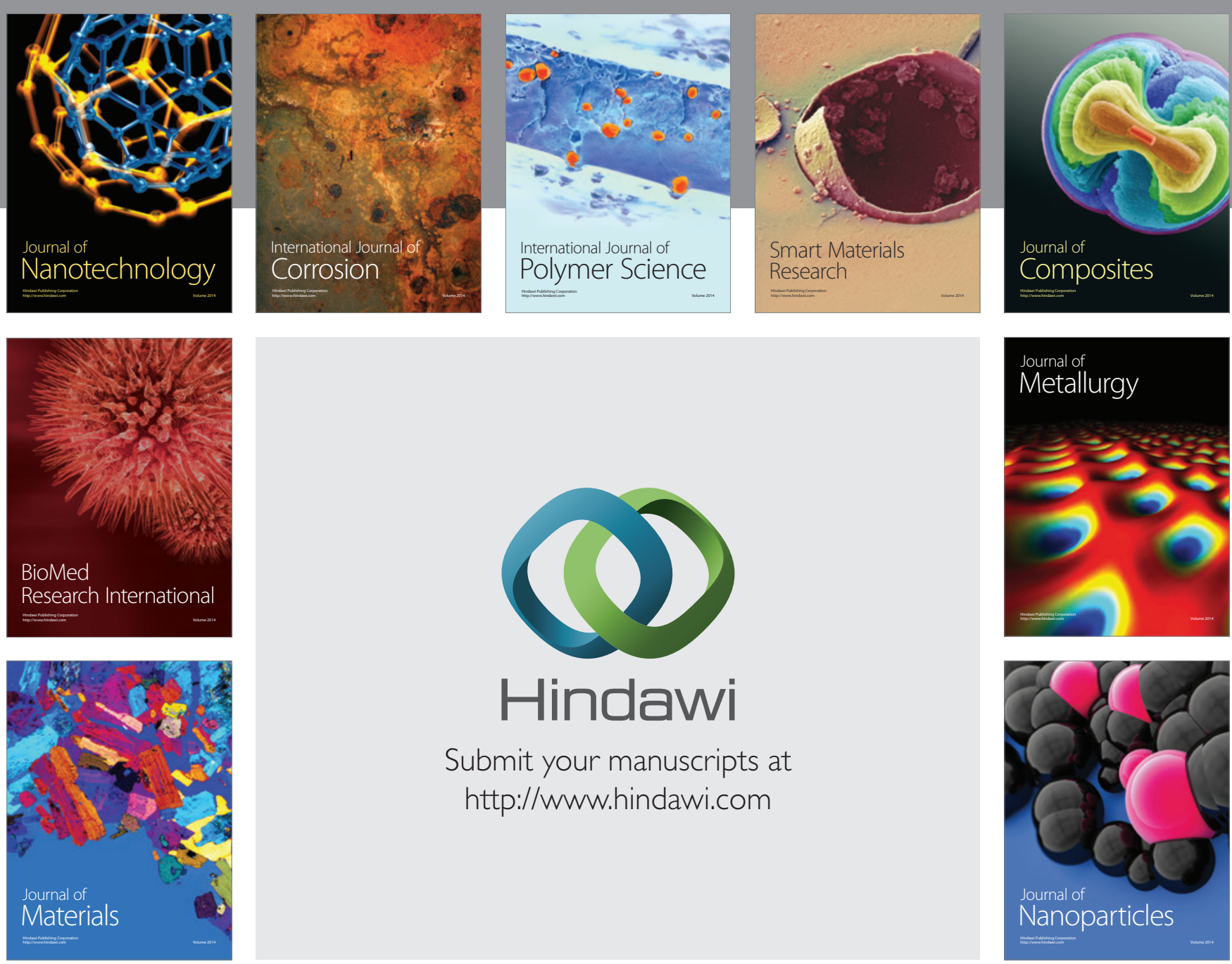

Submit your manuscripts at http://www.hindawi.com
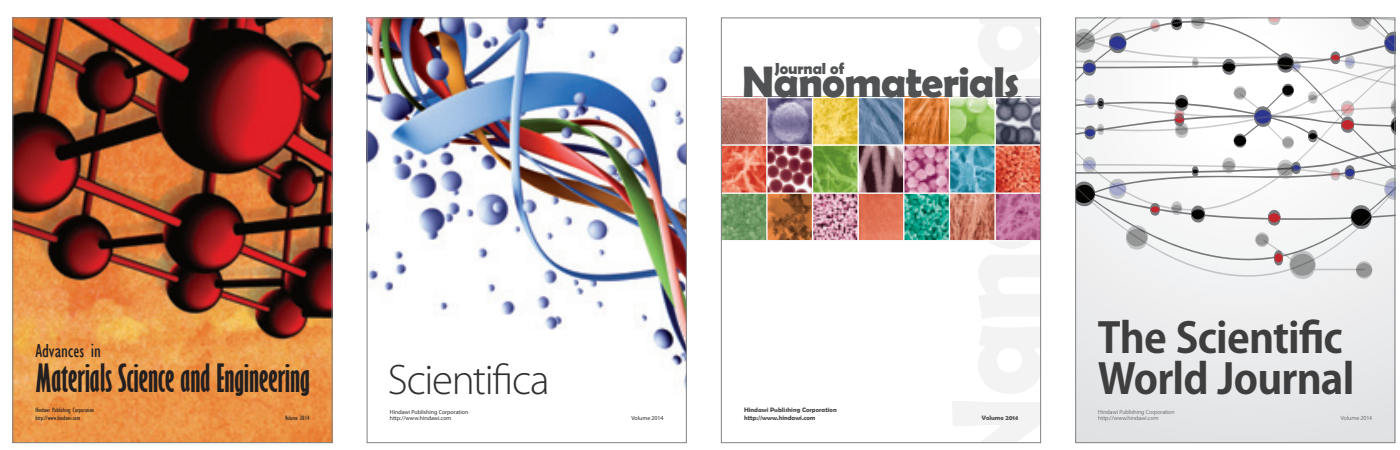

\section{The Scientific World Journal}
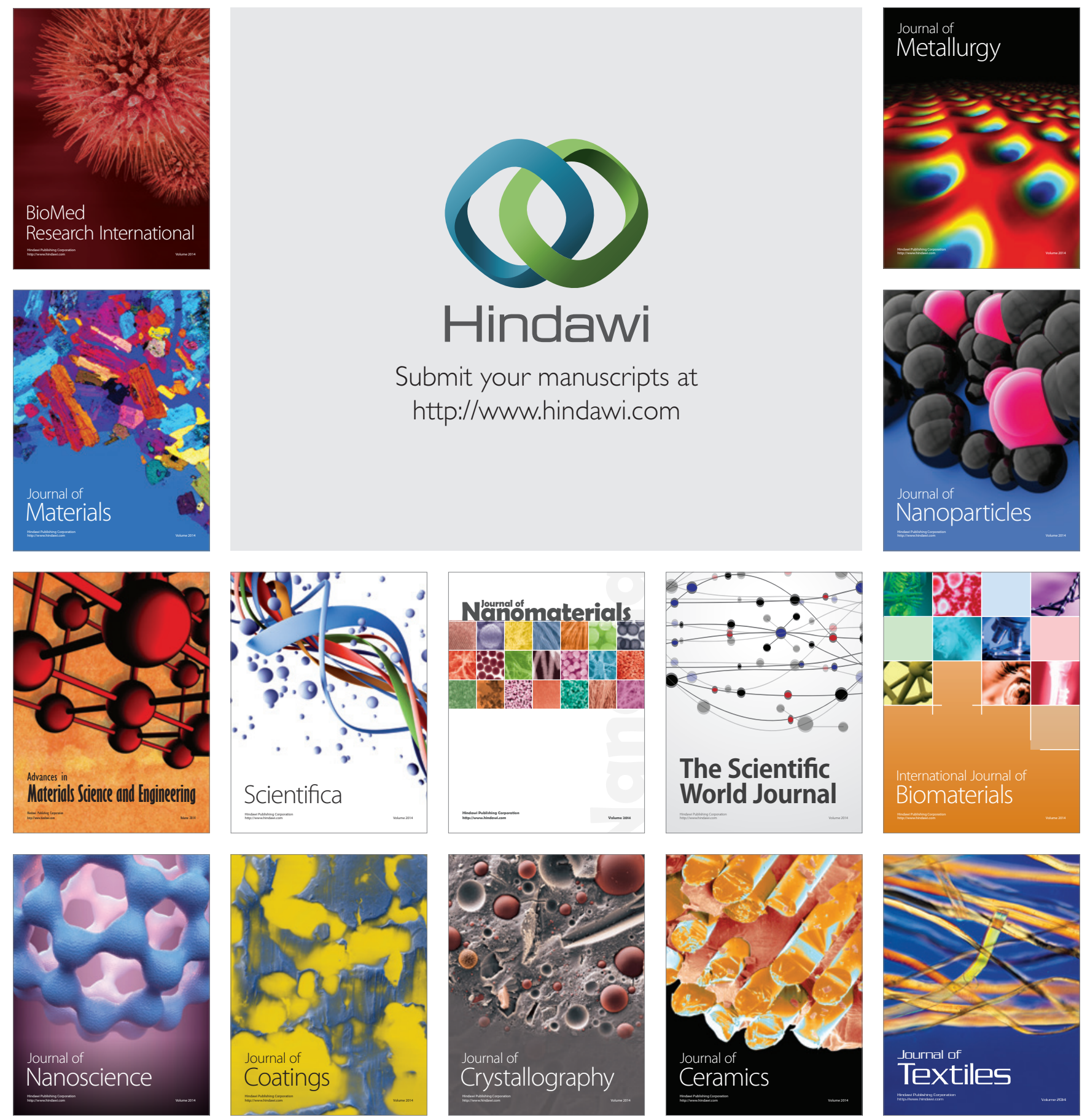